\title{
An Adaptive ISMA-DS/CDMA MAC Protocol for Third-Generation Mobile Communications Systems
}

\author{
Jordi Pérez-Romero, Student Member, IEEE, Ramón Agustí, Member, IEEE, and Oriol Sallent, Member, IEEE
}

\begin{abstract}
In this paper, an inhibit sense multiple access-direct sequence/code division multiple access (ISMA-DS/CDMA) medium access control protocol for a packet transmission mobile radio network is presented. The main feature of this protocol is its ability to retain the inherent flexibility of random access protocols while at the same time reducing to some extent the randomness in the access in order to increase the system capacity. In this framework, the protocol is presented together with some adaptive mechanisms that improve the protocol performance by means of regulating the access and varying the transmission bit rate according to the channel load that is broadcast by the base station. As a result, an adaptive bit rate algorithm is presented that reaches a throughput value close to the theoretical maximum.
\end{abstract}

Index Terms-Access protocols, code division multiaccess, packet radio.

\section{INTRODUCTION}

$\mathbf{R}$ ESEARCH into efficient medium access control (MAC) protocols in a wireless environment gains added interest in light of the fact that third-generation mobile communication systems will be focused on providing wireless access to different kinds of multimedia services, thus needing to handle traffic sources of very different statistical nature with similar capabilities to those of the wired backbone network. In this context, direct sequence/code division multiple access (DS/CDMA) packet transmission networks play an important role thanks to their inherent statistical multiplexing capability.

The main objective of medium access protocols is to guarantee efficient access to a set of shared resources, which strongly depend on the multiple access technique being used. This paper will focus on the DS/CDMA technique, as it is emerging as the predominant multiple access scheme to be used in proposals such as the radio interface defined for universal mobile telecommunication system (UMTS) or cdma2000 (code division multiple access 2000) [1]-[3]. According to this access scheme, in which all the users share the same bandwidth and time slots, the resources to manage become the number of available spreading codes and also the power levels that can be used in order to limit the overall interference level.

For DS/CDMA networks, the most appropriate assignment strategy to manage a set of code sequences will depend on the

Manuscript received March 7, 2000; revised November 14, 2000. This work was supported by CICYT Project TIC 98-684 and under a Grant from the Spanish Educational Ministry.

The authors are with the Department Signal Theory and Communications, Universitat Politècnica de Catalunya (UPC), 1-3, Campus Nord, Edifici D4, Barcelona 08034, Spain (e-mail: jorperez@tsc.upc.es; ramon@tsc.upc.es; oriol@tsc.upc.es).

Publisher Item Identifier S 0018-9545(01)09174-5. service to be provided. For example, in applications such as voice, where users transmit continuous flows of information, the assignment of spreading codes on a per-user basis after an initial setup process can be an efficient strategy. However, this fixed assignment strategy lacks flexibility when dealing with packet-based applications that generate bursty traffic. In such cases, other random access policies become more suitable.

One of the most commonly used random access protocols is slotted ALOHA (S-ALOHA). In a DS/CDMA environment, according to this protocol when a given user needs to transmit a packet, it randomly selects a code sequence from the available set of existing codes in the base station and performs the transmission. This protocol is very flexible for light traffic loads, but as the load increases, the system becomes limited not only by multiuser interference but also by a higher number of collisions in the access. This limitation arises mainly from the fact that users do not have knowledge as to whether or not there are other users transmitting with the selected code sequence.

It is obvious that this type of protocol can be improved by reducing to some extent the randomness in the access. According to this criterion, the inhibit sense multiple access (ISMA) protocol achieves this reduction by broadcasting in the downlink information about the uplink channel. Specifically, the base station indicates the status (busy or free) of the various available spreading code sequences. Hence, users have additional information before attempting to access the system. This protocol was originally designed on a time division multiple access (TDMA) basis as an adaptation of carrier sense multiple access (CSMA) designed to overcome the drawbacks of the radio environment, such as the hidden terminal problem [4], [5]. Some adaptations of this protocol to DS/CDMA are presented in [6]-[8].

The basic behavior of the ISMA-DS/CDMA protocol is shown in Fig. 1 according to the model presented in [7]. Users remain in an idle state while they have no information to be transmitted. Whenever the application layers of a given user require a message to be sent, it is split into fixed length $L$-bit packets. Then, the broadcast channel is listened to in order to determine the available code sequences (i.e., those which are not busy). After this process, one of these codes is randomly selected and the first packet is transmitted. If a collision occurs (i.e., another user has selected the same code sequence in the same time slot) or if the packet is corrupted by multiuser interference, the user will change to the backlogged state and again attempt access in the next time slot by applying a certain probability $p_{r}$. However, if the packet is successfully received and the message contains other packets to be sent, the user will change to the transmission state, the base station will broadcast 


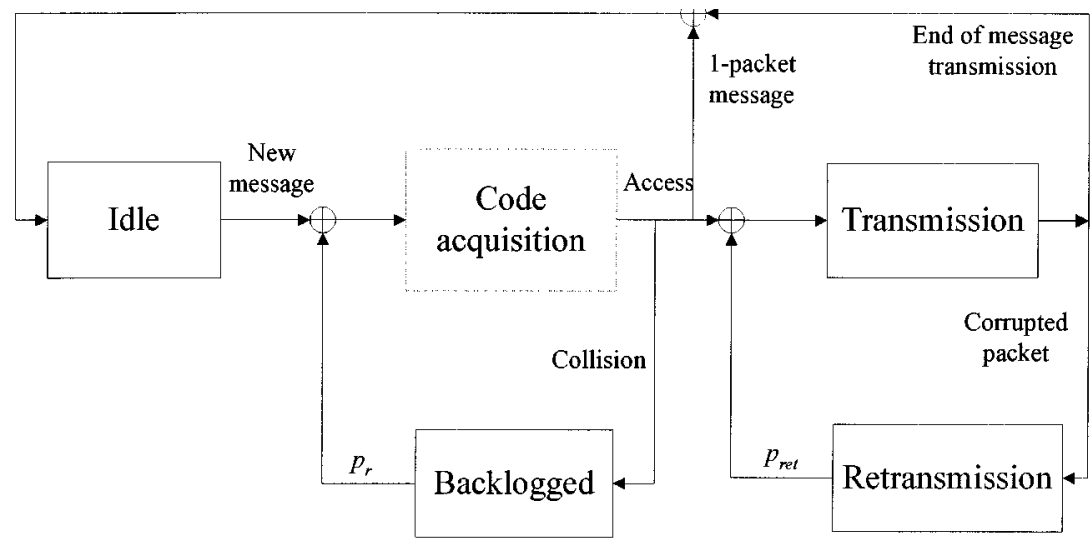

Fig. 1. ISMA-DS/CDMA model.

the selected code as busy, and it will be assigned to the user until the end of its transmission. Then, no other users will be allowed to select that code, so access to that resource is said to be inhibited. However, having acquired a code does not guarantee success in subsequent packet transmissions, mainly due to multiuser interference. So if a received packet contains errors, the user will retain the code, but it will change to the retransmission state and again attempt to transmit the corrupted packet in the next slot by applying a certain probability $p_{\text {ret }}$.

According to this behavior, in [7] a Markov modeling is presented to analyze the performance of the protocol in terms of throughput and message delay, and is validated against simulations. However, this modeling presents some simplifications that are no longer valid when considering the protocol under a realistic frame structure. This paper stems from this basis, and a new ISMA-DS/CDMA MAC protocol is proposed in the context of an frequency division duplex (FDD) frame structure together with some mechanisms that improve its performance based on the knowledge that ISMA-DS/CDMA provides about the system load. Particularly, some strategies are presented regarding the use of adaptive access probabilities and adaptive transmission bit rate algorithms depending on the number of busy code sequences. In this framework, the thresholds mobile station (MS) algorithm (ThMS algorithm) is proposed to vary adaptively the transmission bit rate by taking into account the information provided by the base station as an estimate of the overall interference. This algorithm arises from an evolution of the MS-controlled algorithm (MS algorithm), presented in [9] for an S-ALOHA-DS/CDMA system, by taking advantage of the inherent characteristics of the ISMA-DS/CDMA protocol. Thanks to this feature, ThMS reveals a better reaction capability in the face of changes in the overall system load and also a lower standard deviation in the applied bit rate.

It should be stated that the field of DS/CDMA packet transmission strategies has been recently covered in the definition of the UMTS terrestrial radio access (UTRA) radio interface by third generation partnership project (3GPP) with proposals such as the common packet channel $(\mathrm{CPCH})$ or the random access channel (RACH) [10], [11]. As a matter of fact, there exist some similarities between the proposed ISMA-DS/CDMA protocol and the $\mathrm{CPCH}$ scheme. Consequently, it should be mentioned that the adaptive mechanisms proposed in this paper dealing with the access regulation and the adaptive selection of the appropriate bit rate could be adapted to a scheme like the $\mathrm{CPCH}$ channel.

The rest of the paper is organized as follows. In Section II, the proposed ISMA-DS/CDMA protocol is presented. In Section III, the improvement of the protocol by means of adaptive access probabilities is considered. In Section IV, the adaptive transmission bit rate algorithms MS and ThMS are presented and compared in the ISMA-DS/CDMA context, resulting in a better behavior for the latter. Finally, in Section V, the conclusions are summarized.

\section{ISMA-DS/CDMA PROTOCOL}

The ISMA-DS/CDMA protocol is designed to allow a given number of $N$ registered users working in packet-switched mode to share a set of $K$ spreading codes in a base station thanks to the statistical multiplexing provided by the traffic generation pattern. The considered spreading codes in this work are $T_{f}$ millisecond segments of Gold codes, and each data bit, assuming binary phase shift keying (BPSK) modulation, is spread with a number of chips equal to the spreading factor $S_{f}$. The period of these sequences is then very much higher than the number of chips per bit, and this high period guarantees the existence of a high number of code sequences that can be assigned to the different base stations of the system for the uplink transmission. Additionally, by varying the number of chips per bit, $S_{f}$, different bit rates can be achieved for a user by making use of a single code sequence.

On the other hand, due to the different propagation delays, there will not exist bit level synchronization between the received signals at the base station coming from the different users. Consequently, an asynchronous DS/CDMA system will be assumed in this work.

\section{A. ISMA-DS/CDMA Rules}

The rules that define the proposed ISMA-DS/CDMA protocol are presented in the following, according to Fig. 2 and the basic behavior that has been described in Section I.

1) All the registered users are synchronized with the frame structure of the target base station. It consists of $T_{f}$ mil- 


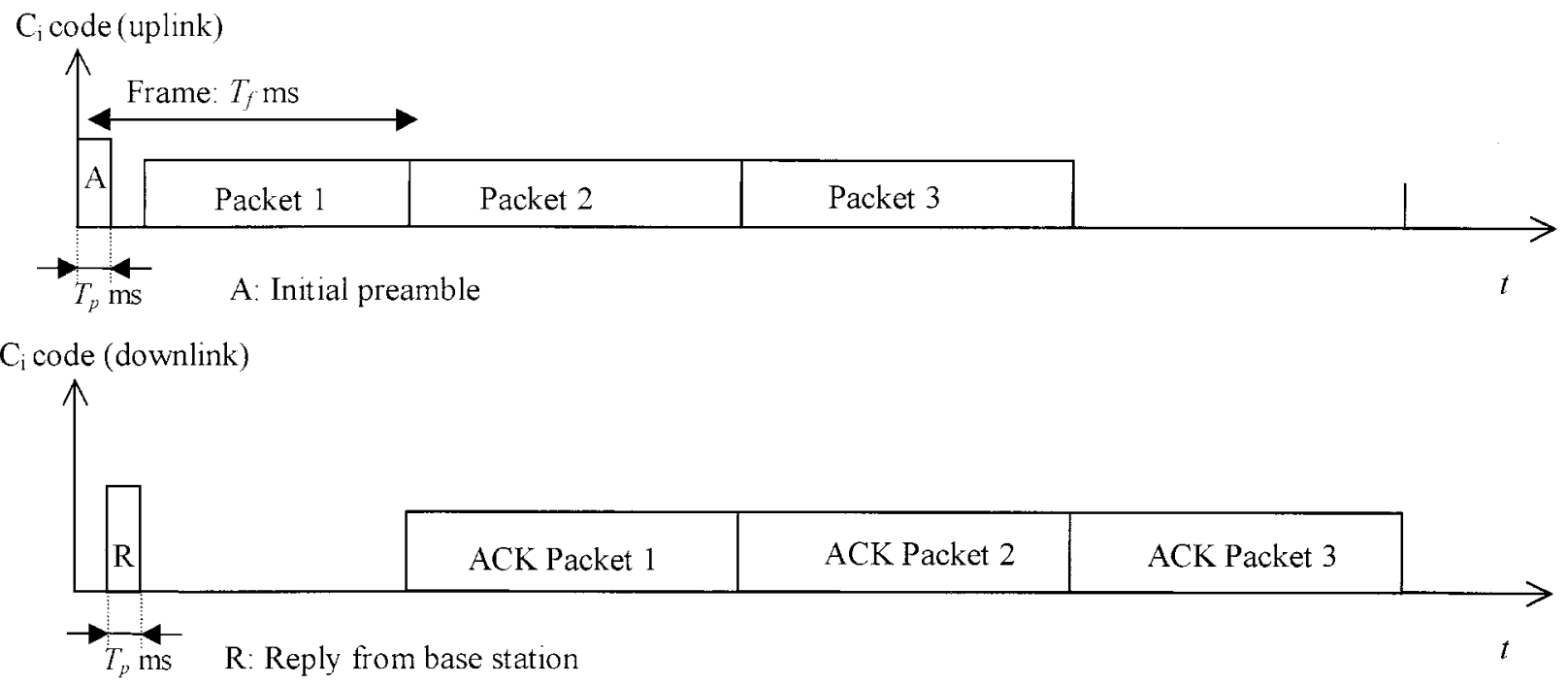

Broadcast (downlink)

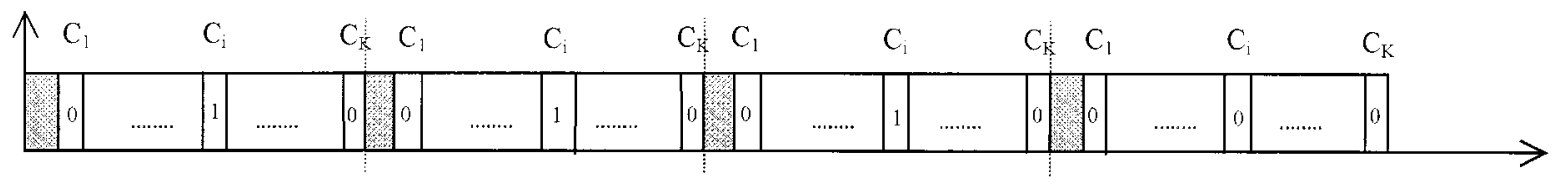

Fig. 2. Frame structure and procedure of the ISMA-DS/CDMA protocol.

lisecond frames which are in turn subdivided into $N_{s}$ time slots, each of them corresponding to a power control period. All the spreading codes of the base station follow the same frame structure.

2) The base station broadcasts on a frame-by-frame basis a channel that contains the status of its $K$ different spreading codes. It requires a single bit per code, where a 1 indicates that the code is busy and a 0 indicates that the code is free. For this analysis, no errors will be assumed in the transmission of this information.

3) Users generate messages that are divided into packets of a fixed integer number of $L$ bits depending on the spreading factor to be used, so that each packet contains the number of bits to be transmitted in each frame. This process must ensure that there is always an integer number of packets per message, by adding zeros if necessary to complete the required number of bits. The generated packets are put into a buffer waiting for transmission.

4) Whenever a message is to be transmitted, the terminal listens to the broadcast channel and determines those spreading codes that are not being used at present. Then, it randomly selects one of them and transmits an initial $T_{p}\left(\ll T_{f}\right)$ millisecond preamble $\mathrm{A}$ by applying an open-loop power control. In addition to other information, a random number is transmitted in this preamble. A certain cyclic redundancy check (CRC) is considered in the transmitted information in order to detect the correct reception of the preamble. In the event of the preamble being correctly received, the base station acknowledges it in the corresponding downlink of the selected code during the next $T_{p}$ millisecond period and replies with the detected random number together with power control indications. The purpose of this random number is to avoid a situation in which two or more users have selected the same code but, due to capture effects, one of the packets has been correctly received. In such a case, only the user whose number corresponds to the number replied by the base station will have acquired the code. This mechanism is similar to the one employed by the global system for mobile communications (GSM) system in the random access procedure [12].

5) After having transmitted the preamble, the mobile terminal listens to the corresponding downlink of the selected code and waits for the base station reply R. To this extent, an error-free return channel will be assumed. If this reply indicates that the code has been correctly acquired (i.e., no collision against another user has occurred and multiuser interference has not degraded the transmission) it continues the transmission for the rest of the frame and the successive frames, while the base station broadcasts the status of the code as busy. On the other hand, if no reply is received, or if the random number does not correspond to the transmitted one, the mobile terminal changes to the backlogged state, and will again attempt access, with probability $p_{r}$, in the subsequent frames. Suitable values for this probability will be discussed later.

The main advantage of this scheme is that it allows detection of a collision just after the first preamble transmission and, consequently, collided users only generate interference to other users at the beginning of a frame, as they abort their transmissions if they do not receive the reply $R$. 
Note in Fig. 2 that the base station can start the broadcast of the spreading codes status (busy or free) only after all the preambles A have been received and thus the base station has been able to determine the new users that have successfully acquired a code. Obviously, this condition does not apply to the rest of system information that can be broadcast in the same channel.

Note also that the packet transmission in the first frame is slightly shorter than the subsequent transmissions. This does not pose any problem in the protocol behavior and just has an implication on how the messages to be transmitted are divided into packets.

6) During the successive frames, the remaining packets are transmitted, and ideal closed-loop power control will be assumed thanks to the dedicated downlink channel and the $N_{s}$ power control periods per packet or frame.

7) The acknowledgment procedure assumes that a packet transmitted in frame $n$ is acknowledged by the base station in frame $n+1$. If a packet is not correctly received due to multiuser interference it will be retransmitted in the subsequent frames with probability $p_{\text {ret }}$, but meanwhile the code will remain busy. No errors are considered in the acknowledgment transmitted by the base station.

8) A bit indicates in each transmitted packet whether or not there are other packets waiting for transmission in the buffer. Then the base station knows when the message transmission finishes and when it has to broadcast the code status as free again. Additionally, the possibility of a user failing before transmitting its last packet has to be taken into account, for example due to loss of coverage. In such a case, the code would remain busy during a certain number of $F_{\max }$ frames without receiving any information from the user and, afterwards, it would be released.

\section{B. System Model Description}

The evaluation of the proposed scheme has been performed by means of system level computer simulations. These simulations consider a given number of registered users in a single cell, each of them generating messages according to a Poisson arrival process. Message length follows an exponential distribution. Both the arrival rate and the mean message length are input parameters of the simulator. Each user follows the transmission rules that have been previously defined for the protocol, by considering an ideal closed loop power control capable of mitigating the channel fading and thus obtaining the same received power for all the users. However, this is no longer true for the transmission of preamble A, in which only an open loop power control can be applied and consequently only the shadowing fading can be mitigated. This point has been simulated by considering a Rayleigh variation in the received preamble.

For each frame, depending on the number of simultaneous transmissions and, consequently, on the overall interference, the simulation computes the number of packets that are successfully received. To this extent, and taking into account that in some instants there exists a mixture of users that apply closed-loop power control with other users that apply an open-loop power control, the model presented in [13] has been considered. Then, the throughput can be computed as the average number of successfully received packets per frame (or equivalently, the mean number of successfully received bits, neglecting the payload introduced by the error detecting code). On the other hand, the delay for a given message can be computed as the difference between the time when the message transmission has been completed (i.e., when all the packets of this message have been successfully received by the base station) and the time when the message arrived into the transmitter buffer.

\section{Maximum Theoretical Throughput Bound of a DS/CDMA Protocol}

In order to evaluate the behavior of a given DS/CDMA MAC protocol, such as the proposed ISMA-DS/CDMA, it is possible to establish a maximum theoretical bound and then see how far is the protocol performance from this maximum. To this extent, let us assume an asynchronous DS/CDMA system with a spreading factor $S_{f}$ (i.e., the ratio of the bit period to the chip period) and $L$ bit packets. Additionally, let us consider that no channel-correcting code is being used and that an ideal closed-loop power control technique is applied in a single-cell scenario. If the code management performed by the MAC protocol were ideal, no collisions would occur in the code acquisition process and thus the system would be limited only by the multiuser interference, which poses a bound for the maximum number of successful simultaneous transmissions inherent to the DS/CDMA technique being used. Particularly, and according to the considered spreading sequences, the Gaussian approximation can be applied to model interference and to determine the bit error probability $p_{b}(x)$ when there are $x$ simultaneous transmissions [14], [15]

$$
p_{b}(x)=\frac{1}{2} \operatorname{erfc}\left(\sqrt{\frac{3 S_{f}}{2(x-1)}}\right)
$$

Then, the maximum throughput (i.e., the maximum number of successful simultaneous transmissions) that could be reached can be calculated by maximizing the following expression where $S$ denotes throughput

$$
\begin{aligned}
S & =x P[\text { correct packet } \mid x \text { simultaneous transmitted packets] } \\
& =x\left(1-p_{b}(x)\right)^{L} .
\end{aligned}
$$

Under this ideal DS/CDMA MAC protocol assumption, the maximization of function (2) corresponds to the maximum throughput value that any protocol could reach under the same assumptions regarding $S_{f}$ and $L$. Hence, it can be used as an upper bound to measure the performance of ISMA-DS/CDMA and determining how far is the behavior of the protocol from the optimum.

Although it can be argued that, for a low number of simultaneous users, the Gaussian approximation is a weak assumption in a DS/CDMA scenario, this is only true for very low bit error rates, which do not have a strong impact on the probability of successfully receiving a packet. Actually, when the number of simultaneous users is small, the bit error rate (BER) is also very small and the probability of successfully receiving a 


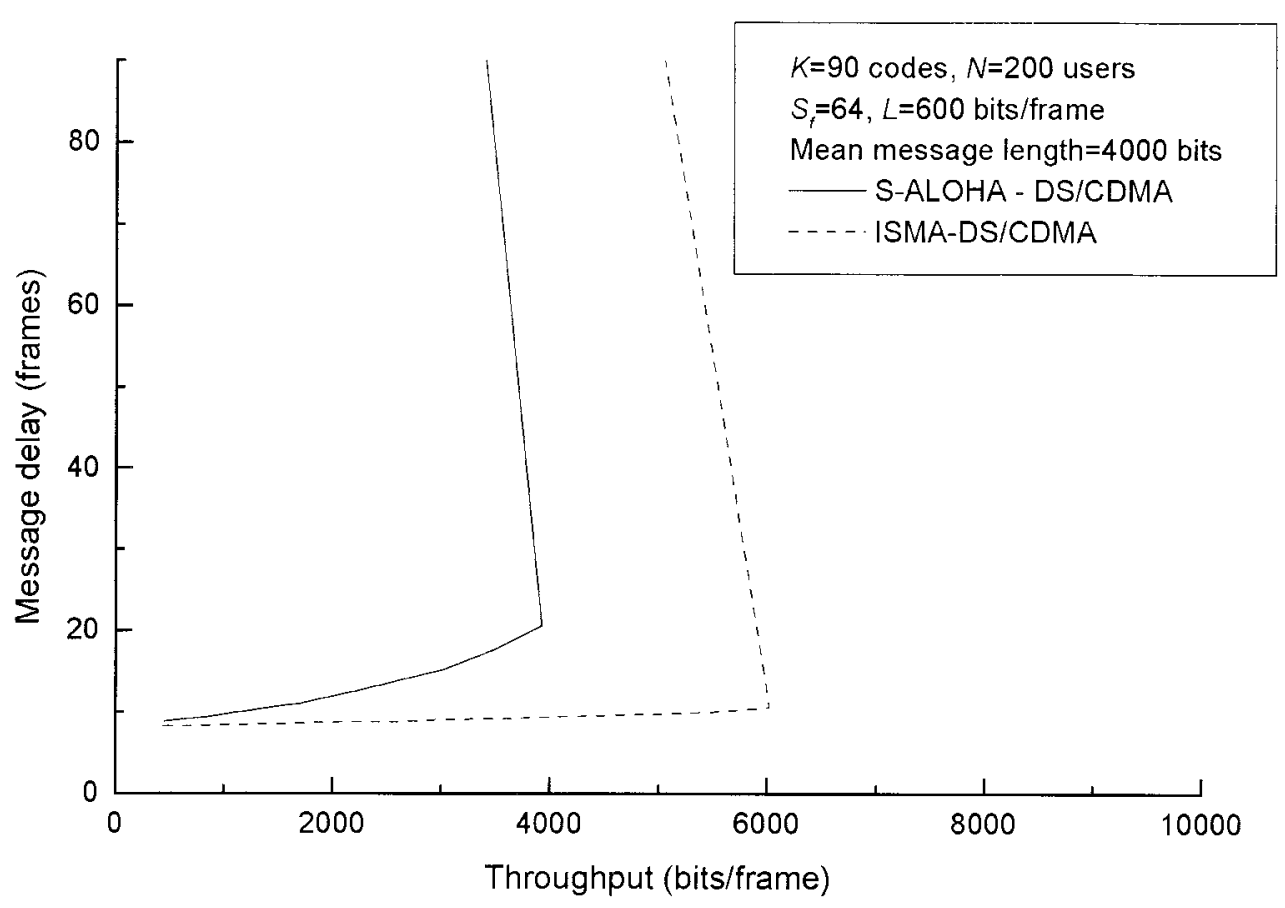

Fig. 3. Comparison between ISMA and S-ALOHA in terms of the throughput versus message delay response.

packet is close to one, regardless of how low the BER is. Consequently, the assumption of considering the Gaussian approximation should not greatly influence the throughput behavior [15].

\section{Comparison With S-ALOHA-DS/CDMA}

In order to show the good behavior of the ISMA-DS/CDMA protocol in a packet transmission environment, the comparison between this protocol and the widely used S-ALOHA-DS/CDMA protocol is presented in the following. For the simulation of S-ALOHA-DS/CDMA, users generate messages according to the same traffic model as has been considered in the ISMA-DS/CDMA case. Then, when they require to access the system they just select one of the available $K$ spreading codes and they transmit a packet on it, the packet duration being equal to one frame. Once they have successfully transmitted the first packet, they will make use of the same code for the remaining packets of the message. However, as there is no broadcast regarding the codes which are being used, a collision with another user that selects the same spreading code can occur in any of the packets of the message. An ideal error-free channel will be assumed for indicating the acknowledgment of the transmitted packets. When a packet is not successfully received, a new access will be tried again in the next frame by applying a certain probability $p_{r}$. Open-loop power control is assumed at the beginning of each packet transmission (i.e., during the first time slot of the frame), while closed-loop power control is considered during the rest of the packet. On the other hand, the throughput and the message delay are computed in the same way as in the ISMA-DS/CDMA case.

Taking into account these considerations, Fig. 3 shows the throughput-message delay response for both protocols when considering $N=200$ users sharing $K=90$ code sequences and an average message length of 4000 bits. We take as a reference the frame structure defined in the UTRA FDD proposal, and thus we assume $T_{f}=10 \mathrm{~ms}$, chip rate of $3.84 \mathrm{Mchips} / \mathrm{s}$ (38 400 chips per frame), $N_{s}=15$ time slots per packet or frame with slot duration of $2 / 3 \mathrm{~ms}$, and $T_{p}=4 / 3 \mathrm{~ms}$ (i.e., the duration of two time slots), together with spreading factor $S_{f}=64$ and packet length $L=600$ bits, corresponding to one of the defined bit rates in this proposal [10]. For both protocols, retransmission of packets that collided is performed with probability $p_{r}=0.3$ in the next frame. For ISMA-DS/CDMA, packet retransmissions once the code has been acquired are performed with probability $p_{\text {ret }}=0.5$. It can be observed that ISMA-DS/CDMA highly outperforms S-ALOHA-DS/CDMA in terms of both throughput and message delay. This is due to the fact that ISMA-DS/CDMA provides users with knowledge about the system status and avoids collisions once a code has been acquired, while in S-ALOHA-DS/CDMA collisions can occur in any packet of the message and thus the number of retransmissions and the delay increase.

On the other hand, the maximization of expression (2) for $S_{f}=64$ and $L=600$ bits gives a maximum theoretical throughput of 14.5 packets/frame or equivalently 8700 bits/frame, while, by looking at Fig. 3, the maximum throughput achieved by the ISMA-DS/CDMA protocol with the specified values for probabilities $p_{r}$ and $p_{\text {ret }}$ is around $6000 \mathrm{bits} /$ frame. This corresponds to an efficiency of around $69 \%$ with respect to the maximum theoretical bound due to the randomness of the protocol in terms of the nonzero collision probability and the effect of probabilities $p_{r}$ and $p_{\text {ret }}$ which have not been optimized yet. Other aspects, such as the lack of perfect power control at the transmission of preambles, also contribute to the difference with the maximum bound. In any case, this efficiency can be increased by making use of the broadcast information regarding the number of busy codes to adaptively vary the 
different probabilities or even the bit rate, as will be discussed in the following two sections. As a matter of fact, the use of this information to improve the protocol behavior is another advantage of ISMA-DS/CDMA over S-ALOHA-DS/CDMA.

\section{ACCESS REgUlATION}

Until now, it has been assumed that all the users attempting to enter the system simply select one available code and transmit on it, while users who fail to gain access try again with some fixed probability $p_{r}$. The purpose of this probability is to regulate to some extent the access into the system when it becomes congested and thus avoid high interference situations. However, this differentiation introduces a degree of unfairness, as new users will gain access more easily than users whose attempt to gain access was not successful, thus punishing the latter in terms of delay. In order to cope with this problem, in this section some strategies to regulate the access fairly, and accordingly, the overall interference level, are presented.

First of all, fairness can be provided by forcing probability $p_{r}$ to be applied not only by backlogged users but also by idle users before entering the system. The main point here, then, is to determine the most suitable value for this probability. Particularly, it should be noted that low values of $p_{r}$ will tend to reduce the number of simultaneous users in the system, thus decreasing the collision probability but increasing the delay, while high values will tend to facilitate the access, thus reducing access delay but also reducing the throughput as the number of simultaneous users will be higher. Hence, a suitable strategy seems to be the selection of an adaptive value depending on the number of simultaneous users (i.e., busy codes) broadcast by the base station. Accordingly, when there are few simultaneous users, high values of $p_{r}$ may be suitable, whereas when the number of simultaneous users increases, it is advisable to reduce or even inhibit new accesses by means of $p_{r}$ values close to zero so that the number of simultaneous users and the overall interference level is kept below a maximum. As a result, $p_{r}$ should decrease from 1 to 0 when the number of busy codes increases from 0 to a given $K_{\max }(\leq K)$ whose value depends on the maximum interference allowed in the system as will be discussed in the following.

According to these constraints, there exist a lot of possible functions for specifying the variation of $p_{r}$ in terms of the number of busy codes $x$ in a given frame. Therefore, this work will be focused only on verifying the benefits of the proposed mechanism for a specific set of functions each of them corresponding to a different general trend of the $p_{r}$ variation, but without attempting to perform an exhaustive search to determine the optimum function. Accordingly, three possible trends to consider for this variation can be the linear dependence, a variation in which $p_{r}(x)$ decreases smoothly for low and medium values of $x$ and abruptly when $x$ is close to $K_{\max }$ [i.e., the second derivative of $p_{r}(x)$ is $p_{r}^{\prime \prime}(x)<0$ ], and finally the opposite variation where $p_{r}(x)$ decreases abruptly when $x$ is low and then it smoothly decreases until reaching $K_{\max }$ [in this case, $p_{r}^{\prime \prime}(x)>0$ ]. Then, three representative functions for these trends would be, respectively:

\section{Linear variation:}

$$
\text { Function 1: } \quad p_{r, 1}(x)=1-\frac{x}{K_{\max }} .
$$

\section{Quadratic variation:}

$$
\begin{array}{ll}
\text { Function 2: } & p_{r, 2}(x)=1-\frac{x^{2}}{K_{\max }^{2}} \\
\text { Function 3: } & p_{r, 3}(x)=\frac{\left(x-K_{\max }\right)^{2}}{K_{\max }^{2}} .
\end{array}
$$

All of these functions are defined only for values of $x$ between 0 and $K_{\max }$, being 0 in all other cases.

The maximum number of allowed simultaneous users $K_{\max }$ needs to be calculated in order to limit the overall multiuser interference present in the system. A possible criterion arises from the maximization of (2) which gives the maximum achievable throughput in an ideal DS/CDMA system and also the number of simultaneous transmissions $x_{\max }$ that provides this maximum throughput. Consequently, it is possible to set $K_{\max }=x_{\max }$ in order that no new accesses are allowed whenever the number of simultaneous users equals $x_{\max }$, as a higher number of simultaneous users would cause the throughput to decrease. Particularly, for the case $S_{f}=64$ and $L=600$ bits, the maximum of (2) is found to be $S_{\max }=14.5$ correct packets/frame, which corresponds to a number of simultaneous transmissions $x_{\max }=16$. Other possible criteria would be to fix $K_{\max }$ depending on a maximum allowable block error rate related to a particular quality of service to be provided.

In Fig. 4, the throughput-message delay response is presented for the three considered functions. As it can be observed, Function 3 presents a poorer performance because it hinders access when the number of simultaneous users is close to $K_{\max }$ by forcing a very low access probability that introduces a higher delay. On the other hand, regarding the comparison between Function 1 and Function 2, differences are small but in general Function 2 allows a lower delay at the expense of a slightly reduced maximum throughput. This is due to the fact that Function 2 presents a higher access probability than the linear Function 1. Therefore, for low and medium load levels, the access delay can be lower with Function 2. Nevertheless, when the number of simultaneous users is close to $K_{\max }$, the higher access probability of Function 2 is reflected on a higher interference level that reduces the maximum achievable throughput.

Although the access is inhibited when there are $K_{\max }$ simultaneous users, as the regulation is performed only in a probabilistic manner, in certain situations it could be possible to have more than $K_{\max }$ users having acquired a code. Note that if there are less than $K_{\max }$ busy codes in the system and thus $p_{r}>0$, as the number of codes $K$ should be higher than $K_{\max }$ to reduce collisions in the code acquisition process [7], there is a nonzero probability that several users succeed in gaining access and hence that there are more than $K_{\max }$ simultaneous users in the system. Therefore, if no additional actions are taken to overcome this issue and limit the number of simultaneous transmissions, a reduction in the throughput could occur.

In order to deal with this problem, a linear backoff strategy can be applied to control the retransmission probability $p_{\text {ret }}$ so 


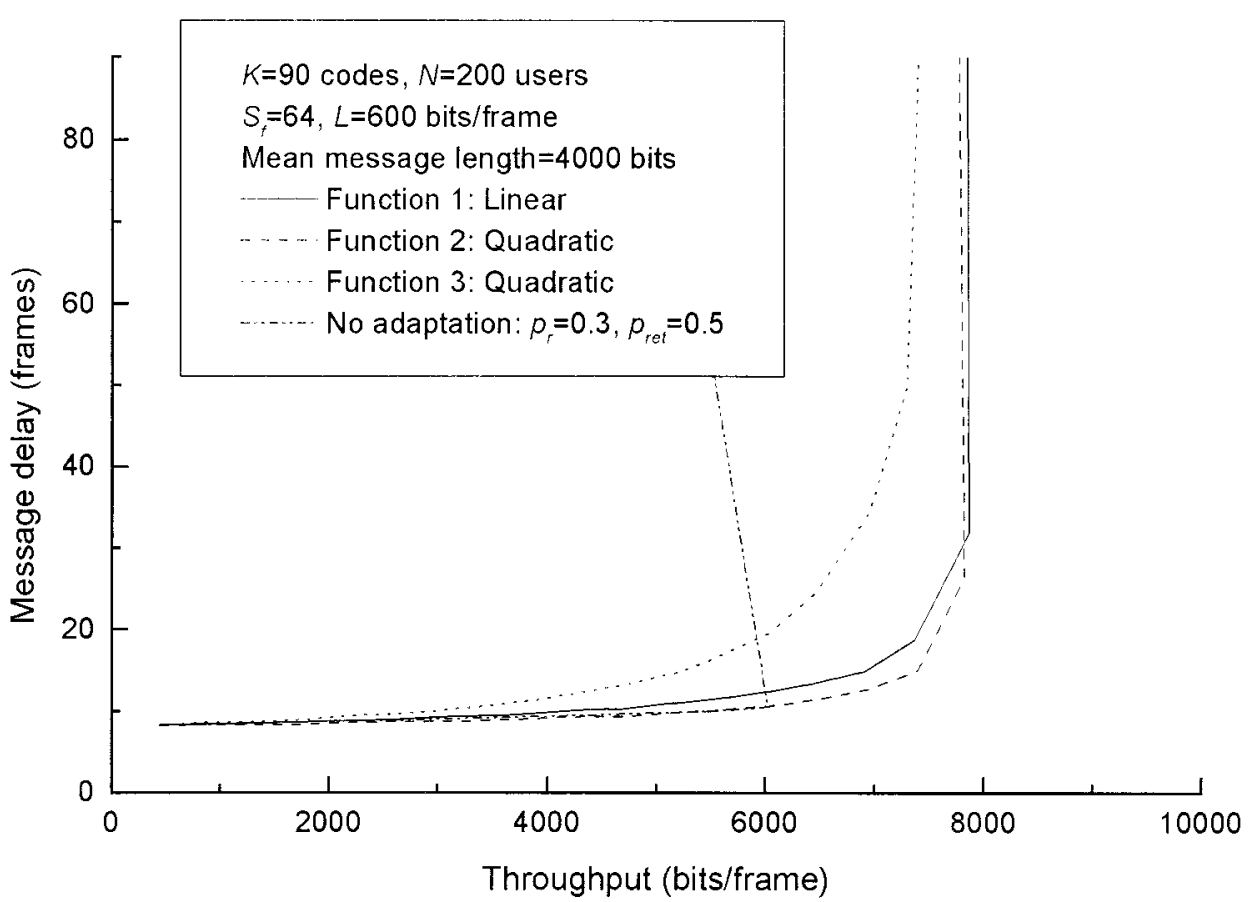

Fig. 4. Throughput versus message delay response for the different variations of $p_{r}$ as a function of the number of busy codes.

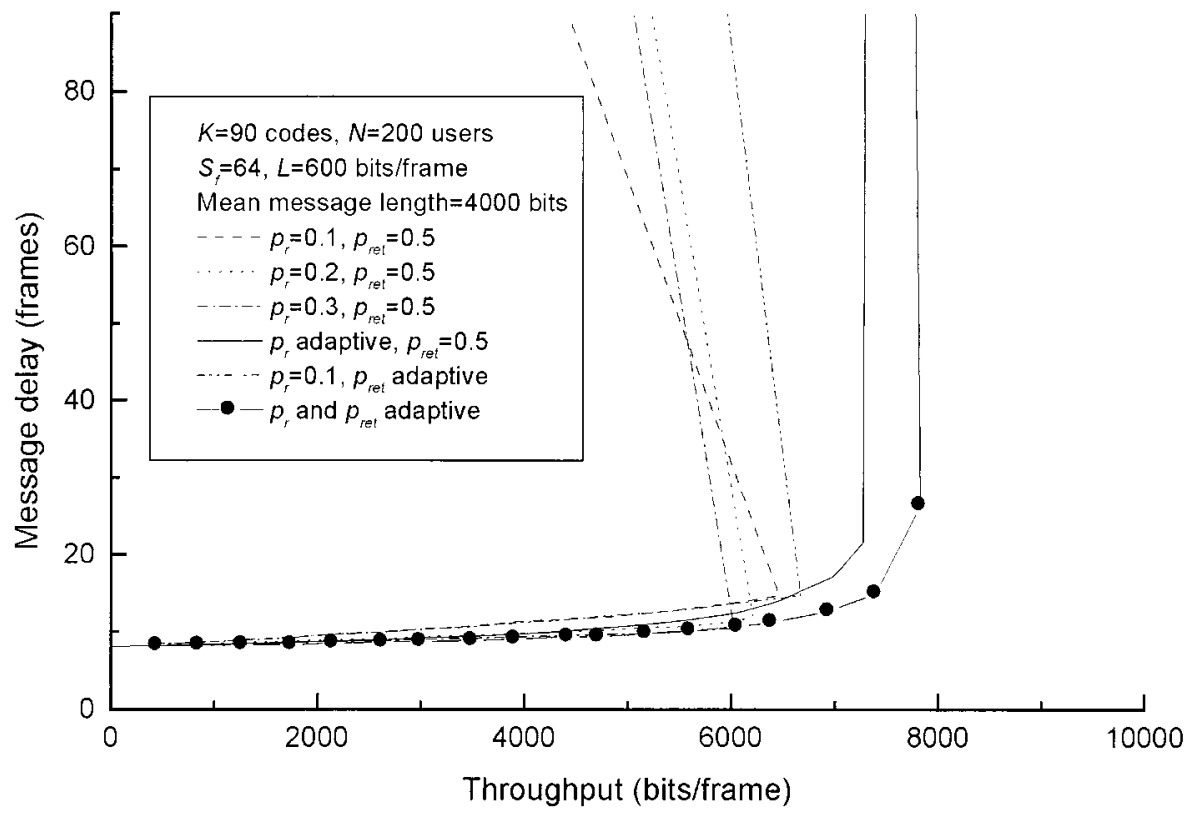

Fig. 5. Throughput versus message delay response of the ISMA-DS/CDMA protocol when using the different adaptive mechanisms.

that users whose packets have not been successfully received put off retransmissions and thus the number of users that are simultaneously transmitting is reduced [16]. Probability $p_{\text {ret }}$ would be decreased as a function of the number of retransmissions of a given packet according to the following relationship:

$$
p_{\text {ret }}(i \text { th retransmission })=\frac{1}{i} .
$$

Fig. 5 presents the results regarding throughput and message delay for ISMA-DS/CDMA when considering fixed $p_{r}$ and $p_{\text {ret }}$ probabilities and when applying the proposed adaptive access scheme for both idle and backlogged users with quadratic Function 2. A significant improvement can be observed when making use of this scheme thanks to the adaptive regulation over the interference level. Likewise, the maximum throughput achieved by the proposed adaptive protocol is around 7800 bits/frame. Then, when comparing it with the maximum theoretical bound of 8700 bits/frame calculated by the maximization of (2), an efficiency in terms of throughput of around 90\% is achieved, higher than in the case of fixed probabilities. Additionally, by looking at the curves in which only one of the probabilities (either $p_{r}$ or $\left.p_{\text {ret }}\right)$ is adapted while the other remains constant, it can be observed how the major benefit of the adaptive mechanism 
TABLE I

CONSIDERED BIT RATES

\begin{tabular}{c|c|c|c|c|c}
\hline Bit rate & $S_{i}$ & $I_{\text {(bits/frame) }}$ & $x_{\max }$ (users) & $S_{\max }$ (packets/frame) & $S_{\max }($ bits/frame $)$ \\
\hline$r$ & 256 & 150 & 86 & 70.5 & 10575 \\
\hline $2 v$ & 128 & 300 & 37 & 31.4 & 9420 \\
\hline $4 v$ & 64 & 600 & 16 & 14.5 & 8700 \\
\hline $8 v$ & 32 & 1200 & 8 & 7.0 & 8400 \\
\hline $16 r$ & 16 & 2400 & 4 & 3.7 & 8880 \\
\hline
\end{tabular}

comes from the adaptation of $p_{r}$ rather than from the adaptation of $p_{\text {ret }}$.

On the other hand, further simulations that we have performed, not shown for the sake of brevity, reveal that the higher the number of code sequences $K$, the higher the benefit that is obtained by means of the adaptive mechanism when compared to the case of fixed probabilities. The reason relays on the fact that if there are a lot of codes, the probability of collision is highly reduced, so that, when fixed probabilities are applied, the system can easily reach a situation where a high number of users have acquired a code depending on the particular values of $p_{r}$ and $p_{\text {ret. }}$. Consequently, if no regulation of the number of transmissions is carried out, the achieved throughput can become very low. Instead, the adaptive mechanism is less sensitive to the number of available codes $K$, as long as this number is higher than $K_{\max }$, because this maximum value actually limits the number of users that have acquired a code, independent of how high $K$ is.

\section{AdAPTIVE TRANSMisSion Bit RATE ALgORITHMS IN ISMA-DS/CDMA}

One of the keys for the flexibility of DS/CDMA is the fact that the transmission bit rate $R_{b}$ can be varied together with the spreading factor $S_{f}$ (i.e., the protection against interference) without modifying the total bandwidth $W$. This comes from the relationship $W=R_{b} \cdot S_{f}$, which means that the bit rate can be increased by a factor of $\alpha$ if the spreading factor is in turn reduced by $\alpha$. Hence, a further improvement can be achieved in the ISMA-DS/CDMA access protocol when applying mechanisms to adapt the transmission rate to channel characteristics. Accordingly, under low-load conditions, users can make use of reduced spreading factors to obtain high bit rates and reduced delays. On the other hand, when the offered load increases, it is better to reduce the bit rate and thus obtain a higher spreading factor that allows more capacity at the expense of tolerating a longer transmission delay.

In the context of S-ALOHA-DS/CDMA, different algorithms have been proposed that deal with the bit rate adaptation depending on the number of erroneous and successful transmissions perceived by a given user, which allows an estimation of the channel load [9]. At this point, it should be stated that a benefit of the ISMA-DS/CDMA centralized operation mode is the intrinsic knowledge that it provides about the channel load by means of the broadcast channel. Hence, this knowledge can be combined with other measurements, such as the number of erroneous and successful transmissions to adaptively decide the suitable transmission bit rate.

In this section, two different adaptive bit rate algorithms for the ISMA-DS/CDMA protocol are addressed. First, the earlier MS-controlled algorithm (MS algorithm) proposed in [9] is considered. Then, the ThMS algorithm is proposed as an improved version of MS in an ISMA-DS/CDMA context that takes advantage of the information about the number of busy codes.

Regarding the system model to evaluate the proposed algorithms, without loss of generality, five of the rates defined in UTRA FDD are considered in this analysis. They are specified in Table I in terms of spreading factors and packet lengths. The number of simultaneous transmissions $x_{\max }$ that maximizes (2) is given for each bit rate together with the corresponding maximum $S_{\max }$. On the other hand, the target received power follows the criterion of having the same energy per bit for all the users, assuming that all of them belong to the same service class. Hence, the received power for a user transmitting at $\alpha v$ bits per second should be $\alpha$ times the received power for a user transmitting at the slowest rate $v$ bits per second.

Another assumption deals with the message generation process. Particularly, it will be considered that the generated messages are divided into packets of length equal to the minimum number of bits that can be transmitted in a frame (i.e., the number of bits corresponding to the lowest possible bit rate). Again, zeros are added if necessary to have an integer number of packets.

\section{A. MS-Controlled Algorithm (MS Algorithm)}

The MS-controlled algorithm (MS algorithm), proposed in [9] for its use together with S-ALOHA-DS/CDMA, is based on varying the bit rate according to the number of consecutive successful or erroneous transmissions for a given packet. The main parameters of this algorithm are $\min _{\text {suc }}$ and $\max _{\text {tr }}$. Specifically, after $\min _{\text {suc }}$ consecutive successful transmissions and if the bit rate is below the maximum one, the rate is multiplied by two as the interference level is assumed to be low enough. On the other hand, after max $_{\mathrm{tr}}$ consecutive erroneous transmissions of a given packet and if the bit rate is above the minimum one, a high interference level is assumed to be in the system and thus the bit rate is divided by two in order to obtain the higher protection provided by the higher spreading factor. This scheme is directly applicable to the proposed ISMA-DS/CDMA protocol 


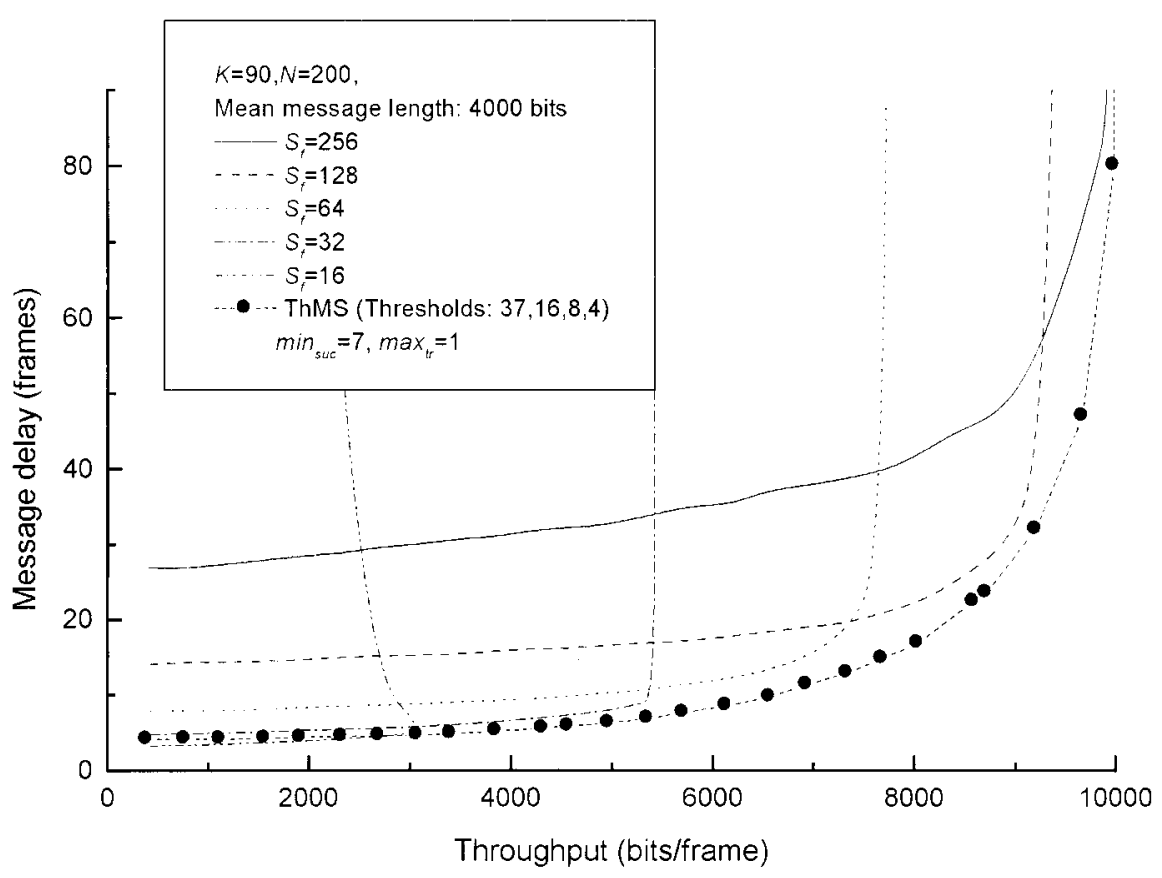

Fig. 6. Throughput versus message delay responses when fixed bit rates are applied and when ThMS algorithm is used.

simply by performing rate adaptations both when the code has been acquired and when the acquisition is being attempted. Note that this scheme is completely decentralized and each mobile terminal selects the corresponding bit rate on its own. The detection of the selected bit rate can be achieved in the base station either by blind detection mechanisms or by indicating it explicitly in a specific field of the transmitted packet.

It should be noted that, since this algorithm was initially designed for a purely decentralized random access protocol such as S-ALOHA, it does not take advantage of the broadcast information to determine the most appropriate bit rate to be used depending on the number of busy codes. As a result, whenever a user starts a message transmission, it will make use of the bit rate that was applied in its last transmitted message, or, in the case of the first access into the system, the lowest bit rate will be applied, with a cautious approach. Therefore, the initial channel load estimation based on previous transmissions can lead to erroneous estimations if message interarrival time is high or if the overall offered load varies with time.

\section{B. Threshold-Based MS Algorithm (ThMS Algorithm)}

For the purpose of overcoming the disadvantages of the MS algorithm, the information provided by the base station can be exploited in order to decide the most appropriate bit rate to start a message transmission. This decision could be taken depending on the number of busy codes $x$, and according to some specific thresholds so that for small values of $x$ high bit rates can be applied and as $x$ increases the bit rate can be decreased accordingly. Let define a set of monotonically decreasing thresholds $\gamma_{i}, i=0,1,2,3,4,5$ with $\gamma_{5}=0$. Then, the initial bit rate $R_{b}$ would be chosen as a function of the number of busy codes $x$ and the slowest bit rate $v$ according to the following relation:

$$
R_{b}=2^{i} v, \quad \text { if } \gamma_{i+1} \leq x<\gamma_{i} \text { for } i=0,1,2,3,4
$$

In order to specify these thresholds, the maximum number of allowed users for each bit rate shown in Table I and derived from the maximization of (2) can be applied. Taking these values would mean that, whenever there are more simultaneous users than the maximum allowed for a given bit rate, a reduction in the initial bit rate would be performed so that in all the cases the bit rate is adapted to the overall interference present in the system. Accordingly, the thresholds are specified as $\gamma_{4}=4, \gamma_{3}=8$, $\gamma_{2}=16, \gamma_{1}=37$.

Similarly, note that according to the access regulation method described in Section III, access is forbidden whenever there are more than $K_{\max }$ busy codes, where $K_{\max }$ is the number of simultaneous users in the system that maximizes the throughput (i.e., 86 according to Table I, corresponding to the case when all the users apply the lowest bit rate). This leads to define $\gamma_{0}=K_{\max }=86$.

Once the transmission has started with the bit rate provided by the thresholds, users will dynamically change their bit rate according to the MS algorithm with parameters min suc $_{\text {and }}$ max $_{\mathrm{tr}}$ depending on the number of consecutive successful and erroneous packet transmissions in the subsequent frames. This mechanism improves the MS algorithm by complementing it with a good initial estimation and thus it avoids the slow starting process to reach the correct bit rate.

In order to study the behavior of these algorithms in an ISMADS/CDMA framework, results have been obtained through simulations when considering $N=200$ registered users sharing a total number of $K=90$ code sequences. Messages are generated according to a Poisson statistic and their length is exponentially distributed with a mean 4000 bits. For both ThMS and MS algorithms we have considered $\max _{\mathrm{tr}}=1$ and $\min _{\mathrm{suc}}=7$, which are the most suitable values according to [9].

Fig. 6 presents the throughput versus message delay response of the ThMS algorithm. The corresponding curves for the dif- 


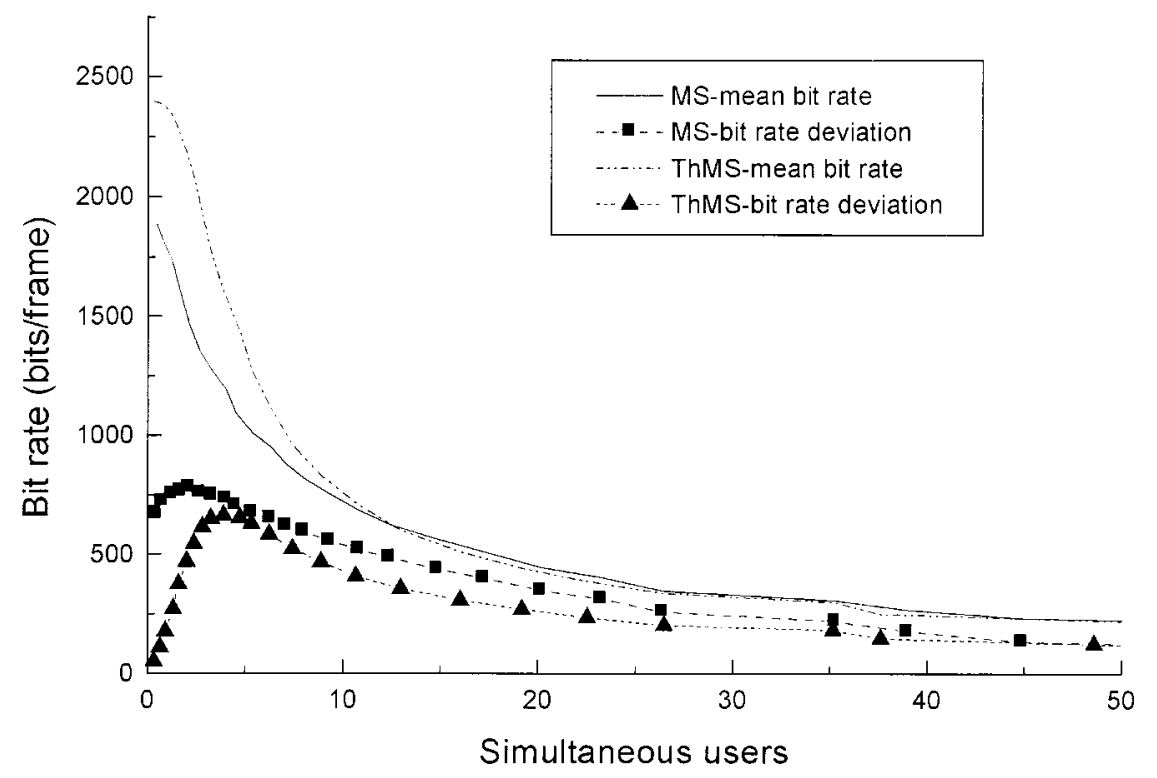

Fig. 7. Mean and standard deviation of the bit rate for the MS and ThMS algorithms as a function of the number of simultaneous transmissions.

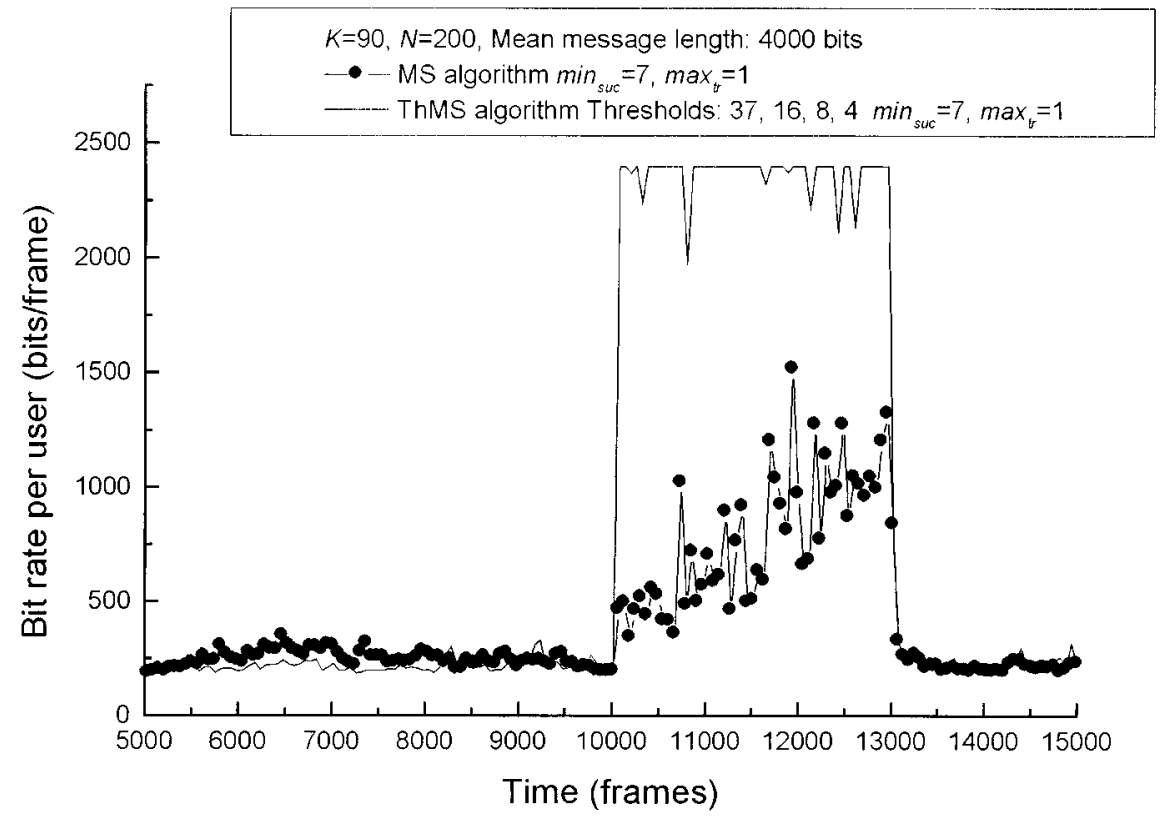

Fig. 8. Comparison between the reaction capability of MS and ThMS algorithms in the face of a change in the overall system load introduced in the simulation between frames 10000 and 13000 .

ferent fixed bit rates are also presented. Regarding these curves, they make use of the adaptive access probability regulation of Section III, with the corresponding values of $K_{\max }$ derived from Table I for each bit rate. A good performance in terms of delay and throughput for both high and low loads can be realized and the response of the ThMS algorithm approximately follows the enveloping of the response for the different fixed bit rates along the whole margin of considered offered loads, as it is supposed to happen with an adaptive bit rate algorithm.

Aiming to compare both algorithms, Fig. 7 shows the mean and the standard deviation of the bit rate applied by each of them as the number of simultaneous users increases. The ThMS algorithm outperforms the MS algorithm for low loads, with a higher mean bit rate that leads to a shorter message delay. The poorer behavior of MS is mainly due to the rough estimation in the initial bit rate, which causes the algorithm to evolve until it reaches the appropriate bit rate, thus leading to a reduced mean bit rate and an increased mean delay. In addition to this, the standard deviation of the MS algorithm is also higher, which reveals that, in practice, there may be users in the system not gaining benefit from the algorithm.

For high loads, both algorithms behave quite similarly in terms of mean delay and throughput. As it can be observed in Fig. 6, the maximum achieved throughput depends on the considered bit rate, so that reduced bit rates allow a higher overall system throughput at the expense of tolerating a higher transmission delay. This aspect reflects the importance of an algorithm, such as ThMS, that is able to select adaptively the 
most appropriate bit rate for each load situation. Note also that the maximum throughput achieved by the algorithm is around $10000 \mathrm{bits} / \mathrm{frame}$, while the maximum theoretical throughput that can be achieved according to the maximization of (2) for the slowest bit rate corresponds to $10575 \mathrm{bits} /$ frame (see Table I). Consequently, the efficiency of the adaptive protocol in terms of throughput is around $94 \%$.

Finally, another important matter to highlight regarding ThMS relays on its faster reaction capability in front of changes in the overall system load, when compared to the MS algorithm. Actually, this is one of the most important features of ThMS, as the bursty traffic involved in a packet transmission network usually suffers from these variations. To illustrate this extent, Fig. 8 presents the instantaneous bit rate achieved by both algorithms in a simulation where a decrease in the overall offered load from 9200 bits/frame to 1500 bits/frame has been introduced between frames 10000 and 13000 by changing accordingly the message generation rate. Each point in the graph corresponds to measuring the bit rate that each user would apply in a given frame and averaging all the measures for this frame.

It can be observed how, thanks to the better estimation of the initial bit rate, the ThMS algorithm is able to easily follow the changes and it switches very quickly to the most appropriate bit rate for each situation, that during the low-load period corresponds to the highest one. On the contrary, the response of MS algorithm to the load reduction requires a slow evolution process due to the initial bit rate estimation based on previous messages transmissions that in some cases do not reflect the real channel traffic load. Consequently, when the reduction in the load arises, the bit rate is progressively increased but in a slow way due to the cautious value of $\min _{\text {suc }}=7$ and the system is not even able to reach the maximum bit rate before the instant when the load increases again, as ThMS does. Although a faster evolution could be obtained by making use of lower values of $\min _{\text {suc }}$, such values would lead to unsuitable bit rate increments which would severely deteriorate performance for high loads [9]. This matter points out another of the problems of the MS algorithm, relaying on a significant sensitivity to the values of parameters $\max _{\mathrm{tr}}$ and $\min _{\text {suc }}$, much higher than the sensitivity that has been observed for the ThMS algorithm when considering different values for these parameters.

\section{CONCLUSION}

In this paper, an adaptive ISMA-DS/CDMA protocol has been proposed. A noticeable improvement in terms of throughput and delay has been obtained when compared to the widely used S-ALOHA-DS/CDMA protocol thanks to the knowledge about the system status provided by the broadcast information. Some strategies designed to improve protocol performance have also been described. First, an access regulation scheme depending on the number of busy code sequences has been proposed in order not to let any user enter the system whenever a maximum number of simultaneous users is reached. This strategy allows an increase in system capacity closer to the theoretical upper bound. Next, two possible adaptive transmission bit rate algorithms dependent on system status have been analyzed on an ISMA-DS/CDMA basis. Particularly, the MS algorithm that was originally proposed for
S-ALOHA-DS/CDMA has been compared with the proposed ThMS algorithm, that applies a threshold-based strategy to decide the initial bit rate for a message transmission depending on the number of busy codes broadcast by the base station. This mechanism allows a higher reaction capability in the face of variations in the system load and also reflects a lower standard deviation of the bit rate. Consequently, ThMS claims for a better overall behavior when compared with MS. The adaptive ISMA-DS/CDMA with ThMS has revealed an efficiency in terms of the maximum theoretical throughput of around $94 \%$.

\section{REFERENCES}

[1] E. Dahlman, P. Beming, J. Knutsson, F. Ovesjö, M. Persson, and C. Roobol, "WCDMA-The radio interface for future mobile multimedia communications," IEEE Trans. Veh. Technol., vol. 47, pp. 1105-1118, Nov. 1998.

[2] E. Dahlman, B. Gudmundson, M. Nilsson, and J. Sköld, "UMTS/IMT-2000 based on wideband CDMA," IEEE Commun. Mag., pp. 70-80, Sept. 1998.

[3] D. N. Knisely, S. Kumar, S. Laha, and S. Nanda, "Evolution of wireless data services: IS-95 to cdma2000," IEEE Commun. Mag., vol. 36, pp. 140-149, Oct. 1998.

[4] K. J. Zdunek, D. R. Ucci, and J. L. LoCicero, "Packet radio performance of inhibit sense Multiple access with capture," IEEE Trans. Commun., vol. 45, pp. 164-167, Feb. 1997.

[5] R. Prasad, "Performance analysis of mobile packet radio networks in real channels with inhibit-sense multiple access," Proc. Inst. Elelc. Eng. $-I$, vol. 138 , no. 5, pp. 458-464, Oct. 1991.

[6] H. van Roosmalen, J. Nijhof, and R. Prasad, "Performance analysis of a hybrid CDMA/ISMA protocol for indoor wireless computer communications," IEEE J. Select. Areas Commun., vol. 12, pp. 909-916, June 1994.

[7] J. Pérez-Romero, R. Agustí, and O. Sallent, "Performance of an ISMA CDMA packet data network," in Proc. VTC in Fall, Amsterdam, The Netherlands, Sept. 1999, pp. 2865-2869.

[8] A. Chockalingam, W. Xu, and L. B. Milstein, "Performance of a multichannel packet CDMA protocol in a fading environment," in Proc. VTC Congr., Phoenix, AZ, 1997.

[9] O. Sallent and R. Agustí, "A proposal for an adaptive S-ALOHA access system for a mobile CDMA environment," IEEE Trans. Veh. Technol., vol. 47, pp. 977-986, Aug. 1998.

[10] 3GPP Technical Specification Release 1999, "Physical channels and mapping of transport channels onto physical channels (FDD)," 3G TS 25.211 v3.3.0, June 2000.

[11] 3GPP Technical Specification Release 1999, "Spreading and modulation (FDD)," 3G TS 25.213 v3.3.0, June 2000.

[12] M. Mouly and M.-B. Pautet, The GSM System for Mobile Communications. Palaiseau, France: Ed. Cell Syst., 1992.

[13] J. Pérez-Romero, L. G. Alonso, and R. Agustí, "Average block error probability in the reverse link of a packet DS/CDMA system under Rayleigh fading channel conditions," IEEE Commun. Lett., vol. 4, pp. 116-118, Apr. 2000.

[14] M. B. Pursley, "Performance evaluation for phase-coded spread spectrum multiple-access communication-Part I: System analysis," IEEE Trans. Commun., vol. COM-25, pp. 795-799, Aug. 1977.

[15] R. K. Morrow, Jr. and J. S. Lehnert, "Packet throughput in slotted ALOHA DS/SSMA radio systems with random signature sequences," IEEE Trans. Commun., vol. 40, pp. 1223-1230, July 1992.

[16] D. Bertsekas and R. Gallagher, Data Networks, 2nd ed. Englewood Cliffs, NJ: Prentice-Hall, 1992.

Jordi Pérez-Romero (S'98) was born in Barcelona, Spain, in 1974. He received the Engineer degree in telecommunications from the Escola Tècnica Superior d'Enginyeria de Telecomunicació, the Universitat Politècnica de Catalunya (UPC), in 1997. He received the Ph.D. degree from he Radio Communications Group in the Department of Signal Theory and Communications at the UPC in April 2001.

$\mathrm{He}$ is currently an Assistant Professor in the field of radio communications. He has been involved in different European Projects and his main research areas are packet transmission mechanisms and radio resource management strategies for CDMA mobile communications networks. 
Ramón Agustí (M'78) was born in Riba-roja d'Ebre (Tarragona), Spain, on August 15, 1951. He received the Engineer of Telecommunications degree from the Universidad Politécnica de Madrid, Spain, in 1973, and the Ph.D. degree from the Universitat Politècnica de Catalunya, Spain, 1978.

In 1973, he joined the Escola Tècnica Superior d'Enginyers de Telecomunicació de Barcelona, Spain, where he became Full Professor in 1987. After graduation, he was working in the field of digital communications with particular emphasis on transmission and development aspects in fixed digital radio, both radio relay and mobile communications. In this framework, he spent six month at the Technical University of Turin, Italy, during 1976-1977 and one month per year at the CNET labs (Lannion, France) during the years 1980-1984. For the last fifteen years, he has been mainly concerned with performance analysis, development of planning tools, and equipment for mobile communication systems, and he has published about 100 papers in these areas. He participated in the European program COST 231 (1989-1996, Evolution of Land Mobile Radio and in the COST 259 (Wireless Flexible Personalized Communications) as the Spanish representative delegate. He has also participated in the RACE and ACTS European research programs in the past (ATDMA, MICC, and RAINBOW projects) and in the IST at the present (WINE GLASS and ARROWS projects) as well as in many privately and publicly funded projects. During this time, he has also been advisor of Spanish and Catalonian Governmental agencies (DGTel, CICYT, ANEP, and CIRIT) on issues concerning mobile communications. $\mathrm{He}$ is a member of the editorial board of several scientific international journals. At present, his research interest lies in the area of mobile communications with special emphasis on CDMA systems, packet radio networks, radio resources allocation and QoS

Dr. Agustí received the Catalonia Engineer of the year prize in 1998.
Oriol Sallent (M'98) received the Engineer and Doctor Engineer degrees in telecommunication from the Universitat Politècnica de Catalunya (UPC), Spain, in 1994 and 1997, respectively.

He joined the Escola Tècnica Superior d'Enginyeria de Telecomunicació de Barcelona, where he became Assistant Professor in 1994 and Associate Professor in 1998. His research interests are in the field of mobile communication systems, especially packet radio techniques and spread-spectrum systems.

Dr. Sallent received the Doctorate Award from the Telecommunication Engineer Association of Spain in 1997 for his Ph.D. dissertation on multiple access protocols for CDMA-based systems. 